Supplement of Atmos. Chem. Phys., 21, 6985-6997, 2021

https://doi.org/10.5194/acp-21-6985-2021-supplement

(C) Author(s) 2021. CC BY 4.0 License.

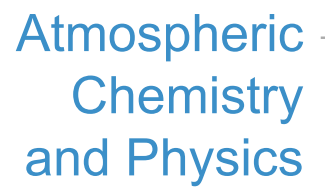

(c) (i)

and Physics

Supplement of

\title{
Robust winter warming over Eurasia under stratospheric sulfate geoengineering - the role of stratospheric dynamics
}

Antara Banerjee et al.

Correspondence to: Antara Banerjee (antara.banerjee@noaa.gov)

The copyright of individual parts of the supplement might differ from the article licence. 


\section{GE08.5}

1

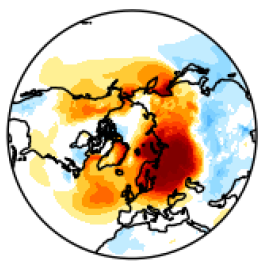

6

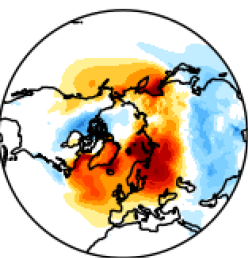

11

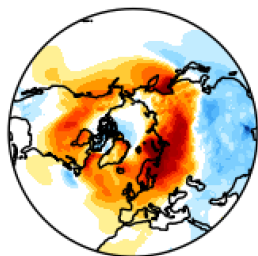

16

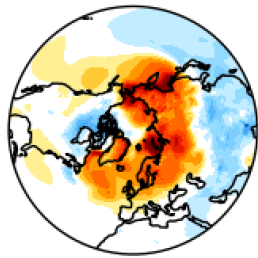

2

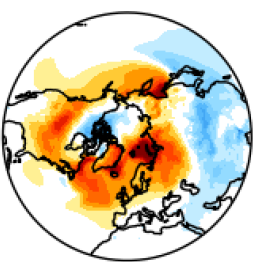

7

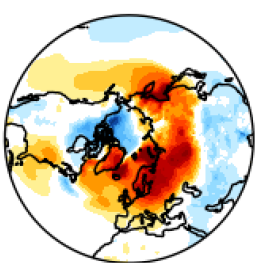

12

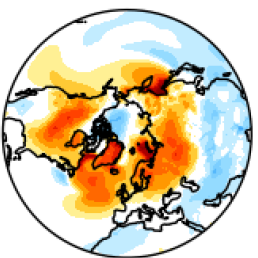

17

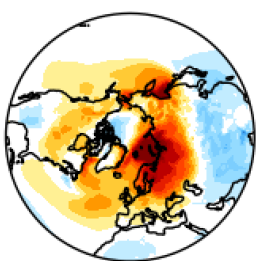

3

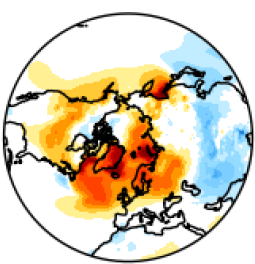

8

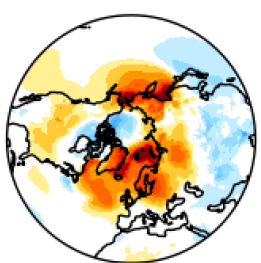

13

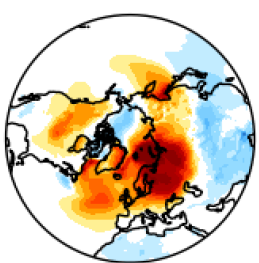

18

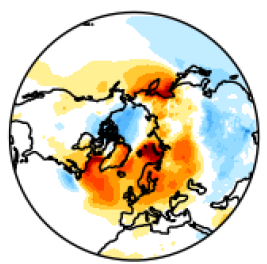

RCP8.5

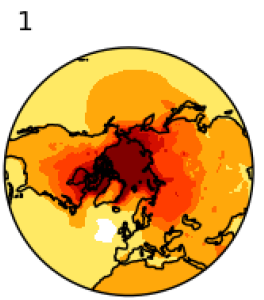

2

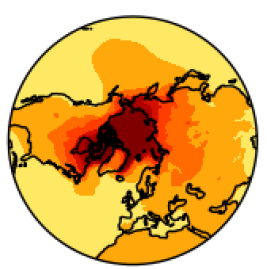

4

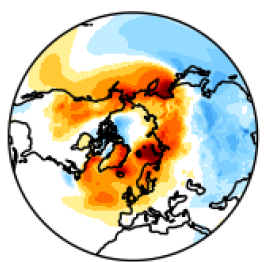

9

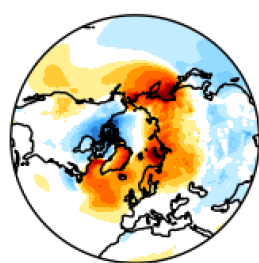

14

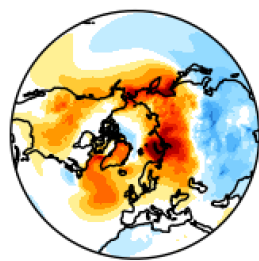

19

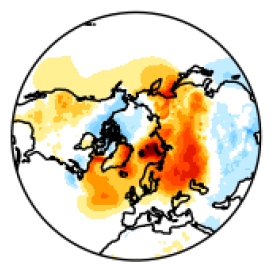

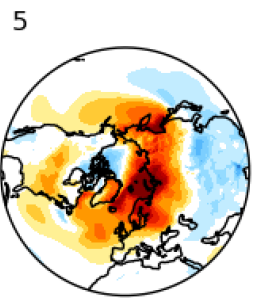

10

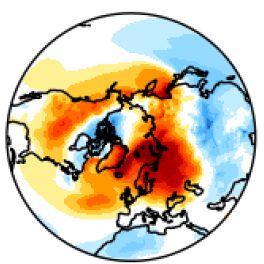

15

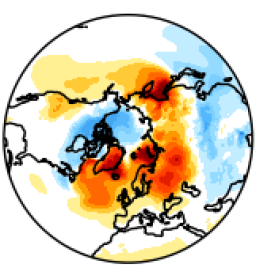

20

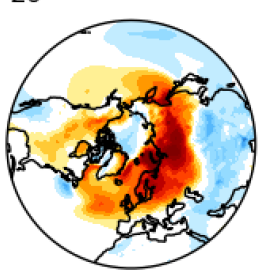

-2.0
-1.6

$-1.2 \stackrel{\text { जn }}{>}$

$0.8 \stackrel{\mathrm{m}}{\mathrm{m}}$

$0.4 \stackrel{\cup}{0}$

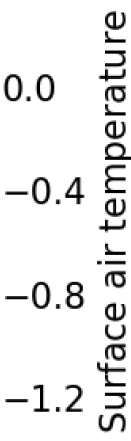

$-1.6$
3

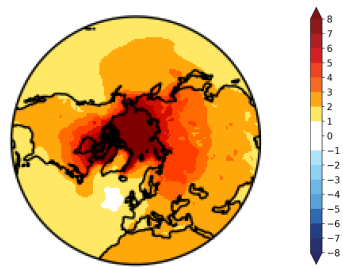

Figure S1. Linear trends over 2020-2095 in near-surface air temperature $\left({ }^{\circ} \mathrm{C}\right.$ per 30 years) in each ensemble member of the GEO8.5 and RCP8.5 experiments. 


\section{GE08.5}

1

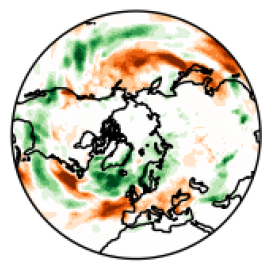

6

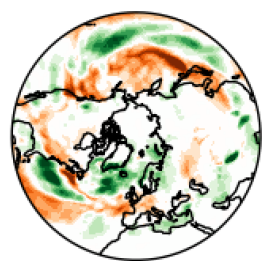

11

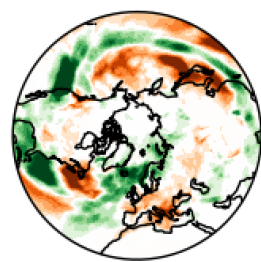

16

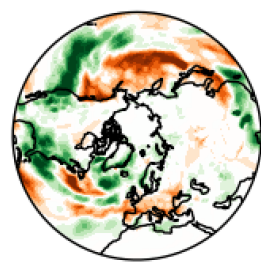

2

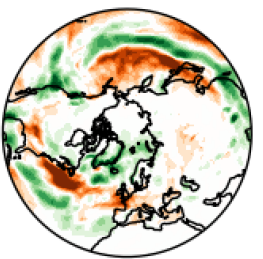

7

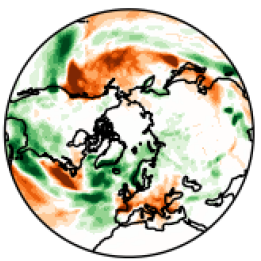

12

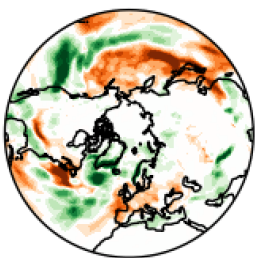

17

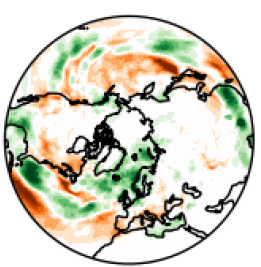

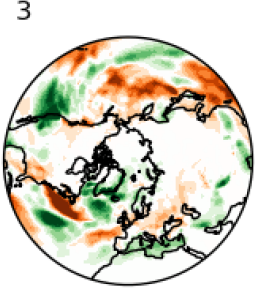

8

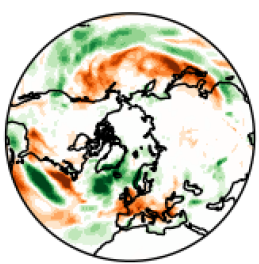

13

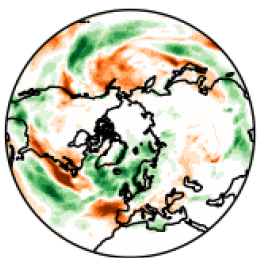

18

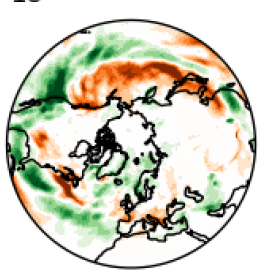

RCP8.5

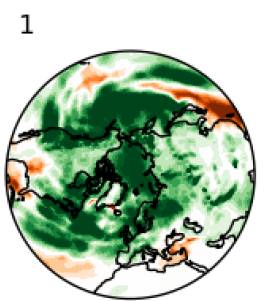

2

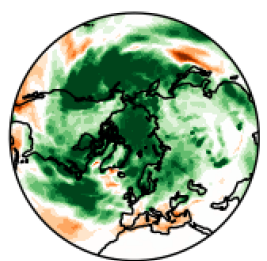

4

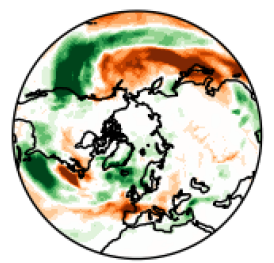

9

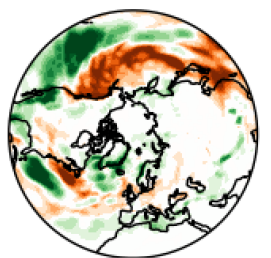

14

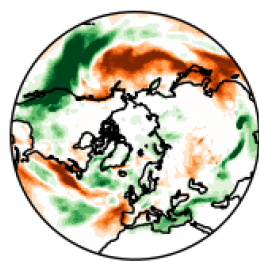

19

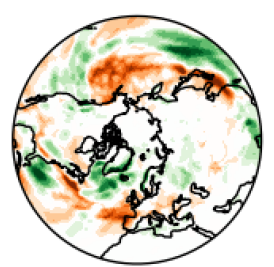

5

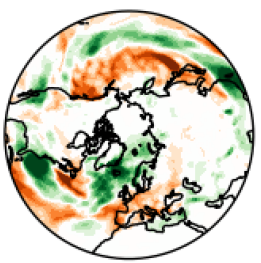

10

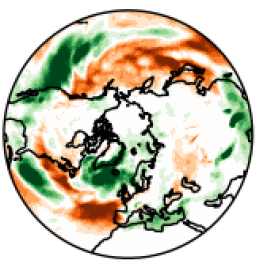

15

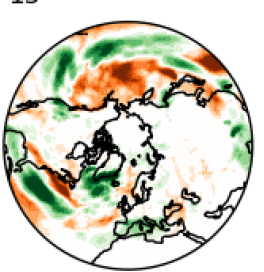

20

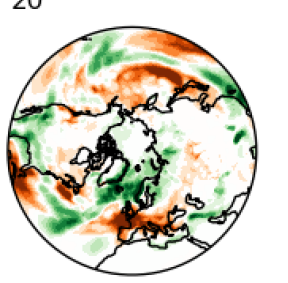

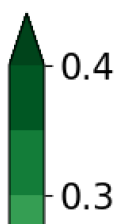

$0.2 \stackrel{\text { ำ }}{\frac{1}{2}}$ $\stackrel{\circ}{m}$ 0.1 ฮั

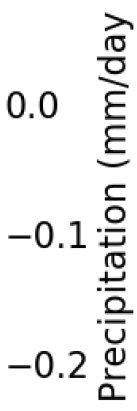
$-0.3$ $-0.4$
3

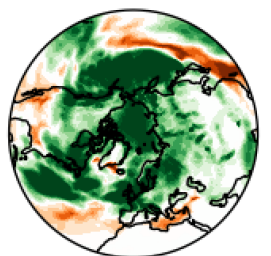

5 Figure S2. Linear trends over 2020-2095 in total precipitation ( $\mathrm{mm} /$ day per 30 years) in each ensemble member of the GEO8.5 and RCP8.5 experiments. 


\section{GEO8.5}

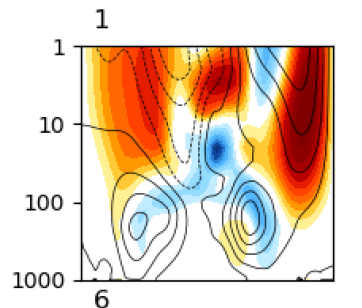

2
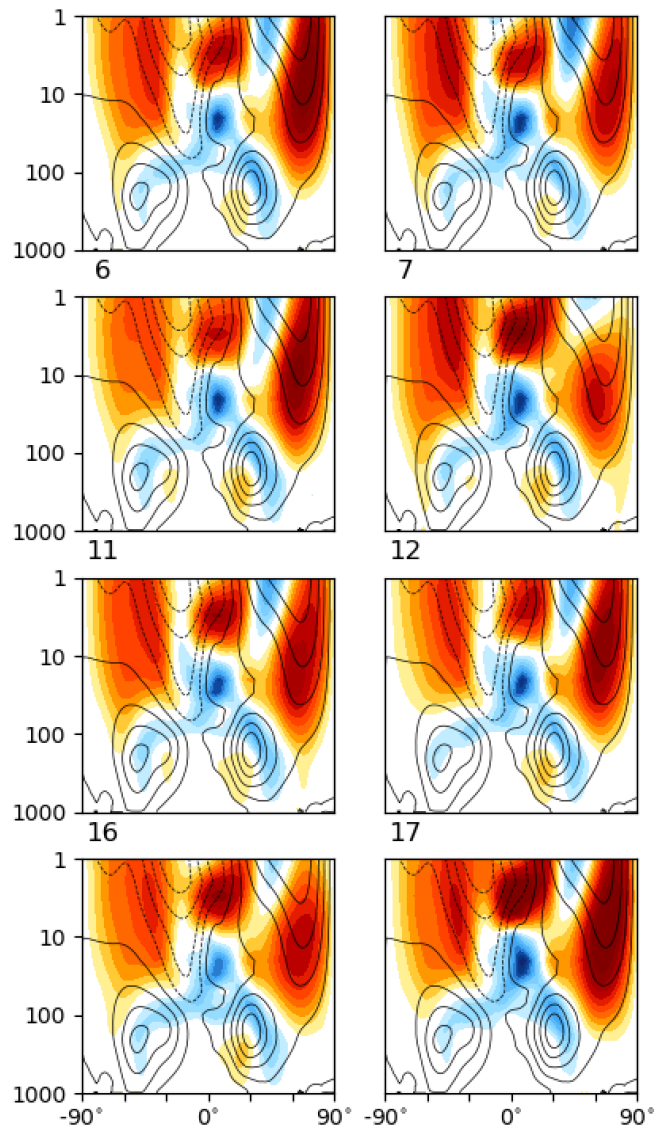

3
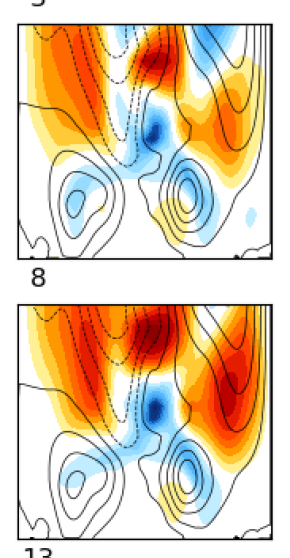

13
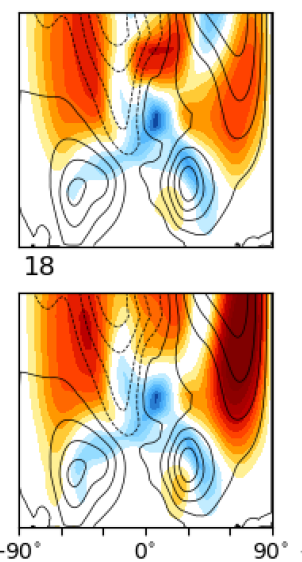

4
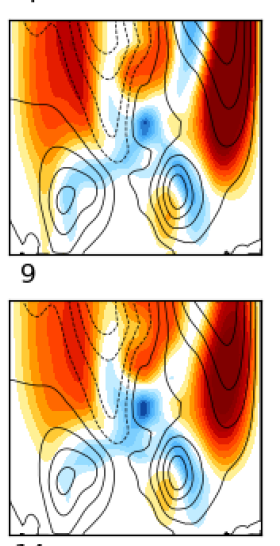

14

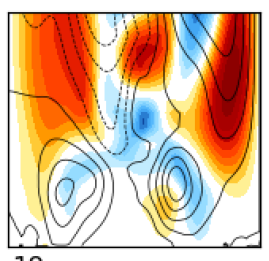

19

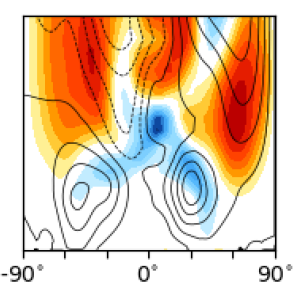

5
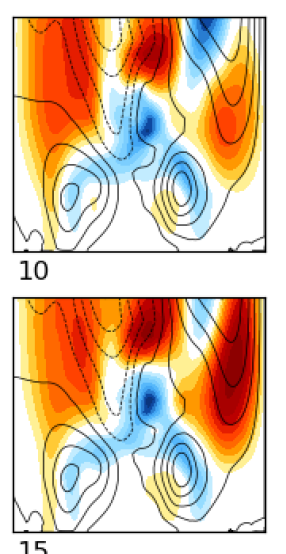

15
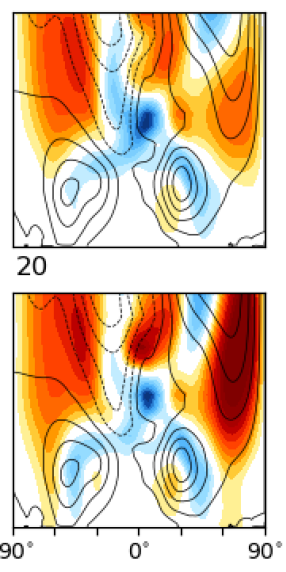

A 5

$-4$

$3 \stackrel{5}{5}$

3 음

$2 \frac{1}{2}$

r

1 ย

I

0

$-1 \frac{\bar{T}}{\frac{1}{\circ}}$

-2 吕

$-3 \frac{\mathscr{E}}{\sigma}$

$-3 \frac{0}{\circ}$
N

\section{RCP8.5}
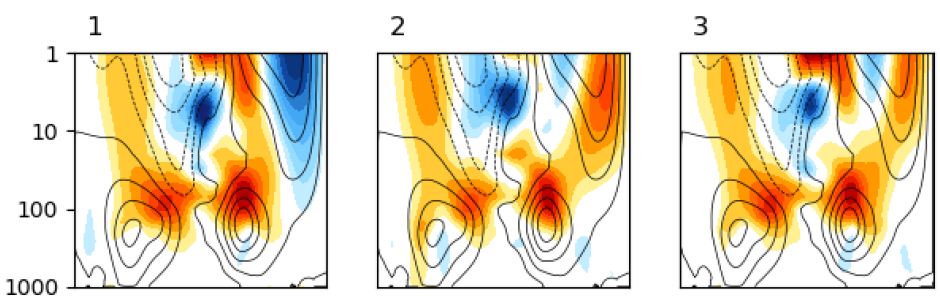

Figure S3. Linear trends over 2020-2095 in zonal mean zonal wind (ms-1 per 30 years) in each ensemble member of the GEO8.5 and RCP8.5 experiments. 


\section{GEO8.5}

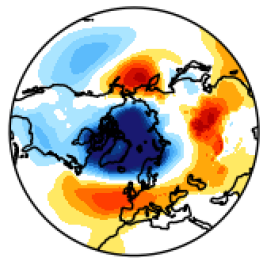

6

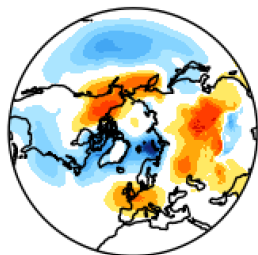

11

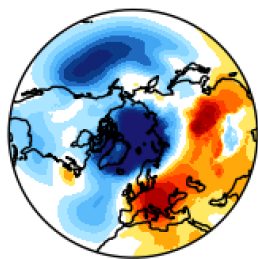

16

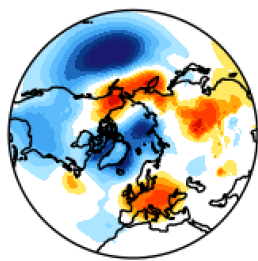

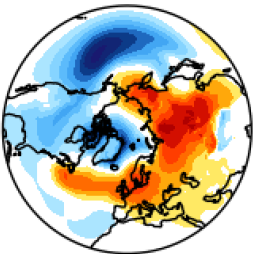

7

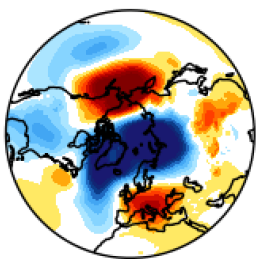

12

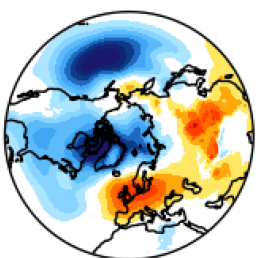

17

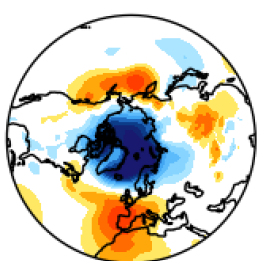

3

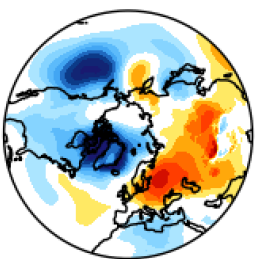

8

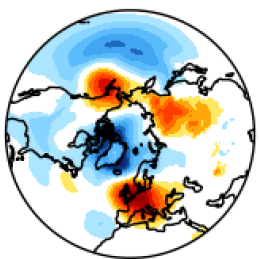

13

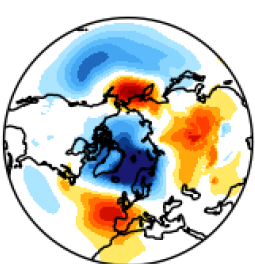

18

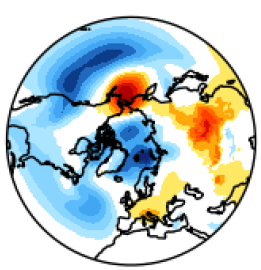

RCP8.5

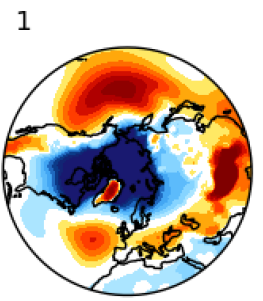
2

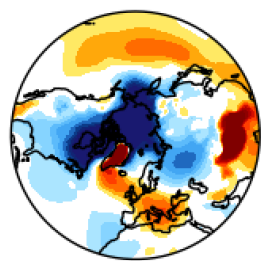

4

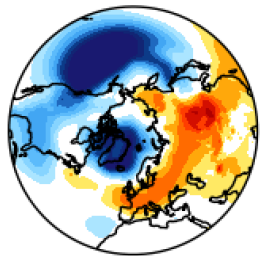

9

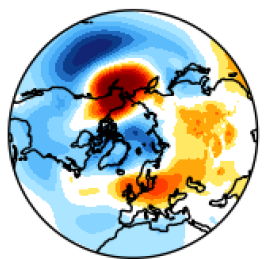

14

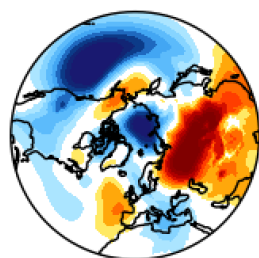

19

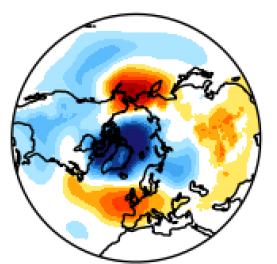

5

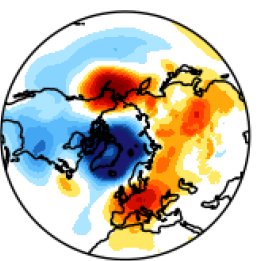

10

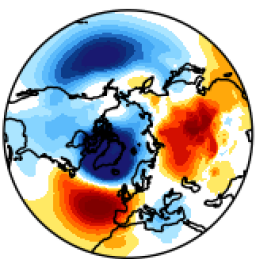

15

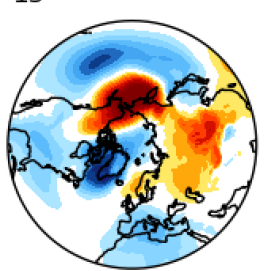

20

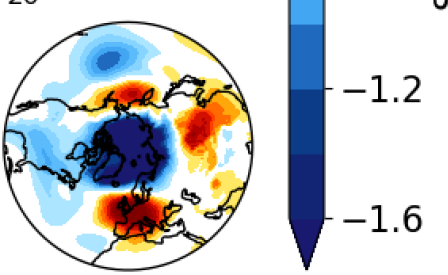

$-1.6$

$0.8 \stackrel{\widehat{n}}{\frac{1}{2}}$ 웅 $0.4 \stackrel{亠}{2}$ 兌 $-0.0$

政 $-0.4 \frac{1}{2}$ 这 $-0.8 \stackrel{\frac{\pi}{4}}{\text { ஸे }}$

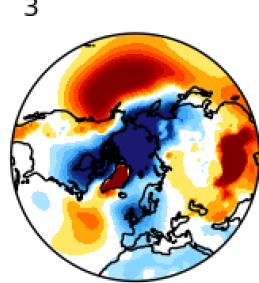

Figure S4. Linear trends over 2020-2095 in sea level pressure (hPa per 30 years) in each ensemble member of the GEO8.5 and RCP8.5 experiments. 